\title{
Symptoms that Required ECG Diagnostics in the Ambulatory Unit of the City Institute for Urgent Medical Care Belgrade Plazinic Djurdja M*, Zivanovic Slavoljub R
}

City Emergency Medical Service, Belgrade djuka031@gmail.com

*Corresponding Author: Plazinic Djurdja M, City Emergency Medical Service, Belgrade.

\begin{abstract}
Introduction: Ambulatory unit of The City Institute for Urgent Medical Care Belgrade (CIFUMCB) is a primary health care institution[1] and is located in Franche D'Epere Street 5, Belgrade. This outpatient clinic operates in day and night shifts. The law [2] stipulates that the CIFUMCB must have an electrocardiography (ECG). The ECG is used at all levels of health care: it is used widely in the pre-hospital phase and at all levels of hospital care for diagnosing and early detection of various disorders, as well as being an integral part of patient triage. At the Community Health Center_depending on needs and accessibility, the general practitioner often records ECG tracings on patients who he is already familiar with. In the outpatient setting of an emergency medicine department, the ECG is often performed for complaints that are not always characteristic for a disease. In contrast to the outpatient clinic of a Community Health Center, patients at the emergency department usually present for the first time, are not familiar to the doctor who examines them, there are no medical records available, and the patients usually do not have medical documentation. Specialists in general and emergency medicine are employed at the CIFUMCB.
\end{abstract}

Keywords: ECG diagnostics, EKG, primary healthcare, City Institute for Urgent medical care

\section{AIM}

To show for which symptoms the ECG was performed at the CIFUMCB outpatient clinic in Belgrade.

\section{MATERIALS AND METHODS}

The electronic database from an outpatient clinic at City Institute for Urgent Medical Care Belgrade (CIFUMCB) was used to gather data from 206 patients examined by one specialist in general practice, for the period from 01-22. January 2015. The presenting complaints were analyzed in patients on whom ECG tracings were recorded; patients may have had more than one complaint listed in the study.

\section{RESULTS}

Out of 206 patients examined, 100 or $48.5 \%$ of them had an ECG performed. In the mentioned sample, there were 59 females and 41 males. Statistical analysis $\left(X^{2}=\right.$ 3.24) indicates that there is no statistically significant difference in sex distribution of the patients, $p>0.05$. The youngest patient on whom ECG was performed was 18 years old and the oldest was 88 years of age.

Table 1. Trouble distribution by groups, on the sample old 100 patients

\begin{tabular}{|c|c|c|c|c|}
\hline $\begin{array}{c}\text { A GROUP OF SYMPTOMS AND } \\
\text { THEIR NUMBER }\end{array}$ & TOTAL NUMBER & \multicolumn{2}{|c|}{$\begin{array}{c}\text { PROBLEMS } \\
\text { (ANAMNESIS) }\end{array}$} & $\begin{array}{c}\text { NUMBER } \\
(\%)\end{array}$ \\
\hline \multirow{5}{*}{ THE FIRST GROUP } & \multirow{5}{*}{60} & \multirow{2}{*}{ chest pain } & constant and dull & 17 \\
\hline & & & pungent and crampy & 8 \\
\hline & & \multicolumn{2}{|r|}{ choking } & 16 \\
\hline & & \multicolumn{2}{|r|}{ cough } & 13 \\
\hline & & \multicolumn{2}{|c|}{ shortness of breath } & 6 \\
\hline
\end{tabular}


Symptoms that Required Ecg Diagnostics in the Ambulatory Unit of the City Institute for Urgent Medical Care Belgrade

\begin{tabular}{|c|c|c|c|}
\hline \multirow{3}{*}{ THE SECONDGROUP } & \multirow{3}{*}{53} & high blood pressure & 31 \\
\hline & & rapid heartbeat & 12 \\
\hline & & irregular heartbeat & 10 \\
\hline \multirow{6}{*}{ THE THIRD GROUP } & \multirow{6}{*}{46} & headache & 15 \\
\hline & & vertigo & 15 \\
\hline & & numbness of hands & 10 \\
\hline & & visual disturbances & 3 \\
\hline & & dizziness & 2 \\
\hline & & insomnia & 1 \\
\hline \multirow{8}{*}{ THE FOURTH GROUP } & \multirow{8}{*}{56} & nausea & 13 \\
\hline & & fever & 11 \\
\hline & & vomiting & 10 \\
\hline & & exhaustion & 7 \\
\hline & & swelter & 6 \\
\hline & & burning sensation in the stomach & 2 \\
\hline & & abdominal bloating and gas & 5 \\
\hline & & $\begin{array}{c}\text { neck stiffness and pain localized in the } \\
\text { left hand }\end{array}$ & 2 \\
\hline
\end{tabular}

The patients' chief complaints were classified according to the most common causes. Complaints in group one suggest diseases of the respiratory system; the second group most often points to diseases of the cardiovascular system; third group indicates disease of the central nervous system, and the fourth group is nonspecific. Some patients had more than one complaint at presentation, which were all allocated into appropriate categories. Regarding other complaints, one patient complained of leg edema as well as cramps in the legs, one of weakness of the right half of the body, one of bleeding from the nose, and one of allergy-urticaria. Out of the total 206 patients, 10 were sent to a secondary or tertiary center on duty, and 12 were sent to a neurologist, surgeon and pulmonologist.

\section{Discussion}

The ECG is a very common and widely used diagnostic method in medicine. Paolo Santos and colleagues showed that the ECG is commonly used in the diagnostic workup by general practitioners [3]. In our study, almost $50 \%$ of the patients at the outpatient clinic at CIFUMCB had an ECG performed, highlighting the frequency of its use. A common reason for performing an ECG in our study was chest pain: blunt or sharp in character, persistent or intermittent, and as stated by P. Santos et al., the ECG is most useful in patients with typical chest pain [ 4]. (see Table 1). In the work of Louise Stone [5], 36.6\% of patients who underwent ECG diagnostics had chest pain. In the work of Rutten FH and associates on the use of ECG at the level of primary health care [6], the most common reason for performing ECG tracings was chest pain, representing $57 \%$ of all complaints. In our research, chest pain accounts for 25 (17 and 8) of all complaints, but if we include those who have other chest complaints, this number increases. (see Table 1). In a Polish study [7], the most common complaint for performing the ECG was chest pain (23\%), which is similar to our results. Chest pain, which can be squeezing, piercing, pressure-like, constant or intermittent, aggravated in certain positions, is a frequent diagnostic challenge in primary health care, and its significance is in confirming or excluding acute ischemic heart disease. CIFUMCB has its own protocol for the treatment of acute coronary syndrome. The ECG is not only done for chest pain, but also for other chest symptoms, such as burning and numbness that may or may not be associated with shortness of breath (etc.), and may also indicate an acute coronary syndrome. This was also discussed in the work: Revised Dutch College of General Practitioners 'practice guideline' Acute coronary syndrome [8]. In our research, from a total of 100 patients, 47 patients had chest complaints, and 
Symptoms that Required Ecg Diagnostics in the Ambulatory Unit of the City Institute for Urgent Medical Care Belgrade

42 had palpitations and collapse. Some patients had more than one complaint at the same time.

In the work of L Stone [9] of 2015, the ECG is said to play the main role in the work-up of chest pain. With regards to chest complaints, shortness of breath - or as the patients themselves put it "choking" or "inability to get air" - was almost as common as chest pain. There were 16 patients with respiratory complaints, which could be placed in the group of patients with chest complaints, and 13 patients with cough. Patients also complained of a rapid and / or irregular heartbeat, which also falls in the category of chest complaints. In the study by FH Rutten et al., it is stated that one of the main reasons for the use of ECG in diagnostics, in addition to chest pain, are collapse and palpitations, accounting for $30 \%$ of all cases [6]. In our study, $10 \%$ of our patients had ECG tracings performed for irregular or raid heartbeat, while fainting accounted for $2 \%$ of complaints. Numbness that propagated left or right along the arm, starting from the shoulder to the wrist, was recorded in 10 patients. (see Table 1)

The most common reason for the use of ECG in diagnostics was elevated blood pressure, which was the presenting complaint in 31 patients. According to the ESH / ESC recommendations, a 12 channel ECG should be a part of the routine examination of a hypertensive patient [10]. Some of the patients who complained of high blood pressure also reported concurrent headaches and other complaints. Why perform the ECG in patients presenting with headaches? For these patients, whether presenting with headache alone, or in association with other complaints, e.g. elevated arterial pressure, the ECG can be a part of the initial assessment [11].

The distribution of complaints indicates that they are most often associated with the respiratory, cardiovascular and central nervous system. (see Table 1). Comparing the above three organ systems, $X^{2}=$ $1.849057 \mathrm{p}>0.05$ showed that there is no statistical difference in the representation of complaints by organ system. Some patients had complaints belonging to several organ systems, i.e. there are "common-in-each group. Analyzing the complaints of our patients - chest pain and rapid or irregular heartbeat, and comparing them to results in previously published papers [5,3], we report similar percentages. A study conducted in Portugal [5] reported $36.6 \%$ of patients reporting chest pain, and $23.3 \%$ complaining of palpitations. In our study, $22 \%$ patients reported of irregular or rapid heartbeat, keeping in mind that one patient could have multiple symptoms at the same time. At the outpatient clinic at CIFUMCB in Belgrade, 15\% of patients complained of faintness or vertigo, and $6 \%$ of difficulty breathing. Research in Portugal [5] recorded almost the same percentages (15\% and 5.7\% respectively). A Polish study, which collected data by telegraphy of the ECG tracings [6], 13\% of patients complained of syncope or vertigo. Another study in Poland [4] mirrors percentages recorded at CIFUMCB, reporting chest pain in $23 \%$ and heart disorders in $18 \%$ of patients as the complaint leading to ECG.

Non-specific complaints that occurred independently or associated with other complaints (mentioned above) were often the presenting complaint. Thus, in the study Managing the consultation with patients with medically unexplained symptoms: a grounded theory study of supervisors and registrars in general practice [4], patients with non-specific complaints often reported to a general practitioner and expected an explanation for their complaints, as well as appropriate medications. A consultation without an ECG being performed could be disconcerting for the patient [4]. In doing the ECG, patients' complaints are often resolved [4], or alternatively the ECG indicate whether or not there is a serious cardiac disorder. In the work of Electrocardiography in primary care; is it useful? [3], before an ECG, it was estimated that 34\% of the patients were to be referred to a cardiologists. After performing an ECG, the percentage of patients referred to cardiology dropped to $20 \%$. In our study, only $10 \%$ of patients were referred to internists for further work-up, showing a direct benefit to alleviating the burden on higher levels of health care, in this case internist.

Often patients want to know not only what is happening to them, but whether it is serious. The ECG often resolves this issue [4], which is often, from the perspective of the physician, the most important to the patient.

\section{CONCLUSION}

In our study, patients who underwent ECG most often presented with complaints that indicate diseases of the respiratory, cardiovascular and central nervous system. The most common presenting complaint 
Symptoms that Required Ecg Diagnostics in the Ambulatory Unit of the City Institute for Urgent Medical Care Belgrade

was elevated blood pressure, followed by chest paindiscomfort in the chest, irregular and rapid heartbeat, headache, or dizziness. Only a small number of these patients were referred to the internists for further diagnosis and treatment.

\section{REFERENCES}

[1] Law od healthcare, "Official Gazzete of RS", No.107/2005. Activity and organization of health services, health services on the primary level, Article 105 Avaliable from http://zso. gov.rs/doc/dom-p/zdr-os/Zakon \%20o\%20 zdravstvenoj\%20zastiti2.pdf citted20.2.2017.

[2] Rules on detailed conditions for doing health services in health institutions and other forms of health services, "Official Gazette of RS", No.43 / 2006. A list of equipment for the medical industry. Avaliable from https://tinyurl.com/ zl9plcd citted 19.2.2017.

[3] Santos P, Pessanha P, Viana M, et all. Accuracy of general practitioners' readings of EKG in primary care. Cent Eur J M. 2014 June; 9(3): 431-436

[4] Santos P, Carlos M, Sa L, Hespanhol A, Couto L. Motives for requesting an electrocardiogram in primary health care. Cien Saude Colet. 2015 May; 20(5) about 5 pages. Internet Avaliable from: http://tinyurl.com/z3hh6qw

[5] Stone L. Managing the consultation with patients with medically unexplained symptoms: a grounded theory study of supervisors and registrars in general practice BMC Family Practice 2014 dec; 15:192

[6] Rutten FH, Kessels AG, Willems FF, Hoes AW. Electrocardiography in primary care; is it useful? Int J Cardiol. 2000 Jul 31; 74(2-3):199-205.

[7] Rekosz J, Gałazkowski R. 12-lead EKG teletransmission in emergency medicine teams' actions. Wiad Lek. 2013; 66(3):219-25.

[8] 8. Bouma M, Rutten FH, Wiersma T, Burgers JS. Revised Dutch College of General Practitioners' practice guideline 'Acute coronary syndrome'. Ned Tijdschr Geneeskd. 2013;157(8):A6006.

[9] Santos P, Martins Ca, Pessanha P, et all. Electrocardiogram and Chest Pain in Real Practice: A Retrospective Cohort Study in Family Practicein Portugal. Research Journal of Medical Sciences 2015; 9: 58-63.

[10] Mancia G, Fagard R, Narkiewicz K, Redón J, et al. 2013 ESH/ESC Guidelines for the management of arterial hypertension: the Task Force for the management of arterial hypertension of the European Society of Hypertension (ESH) and of the European Society of Cardiology (ESC) 2013 Jul; 31(7):1281-357

[11] Forbes RB. Acute Headache. Ulster Med J. 2014 Jan; 83(1): 3-9..

Citation: Plazinic Djurdja M., Zivanovic Slavoljub R. Symptoms that Required Ecg Diagnostics in the Ambulatory Unit of the City Institute for Urgent Medical Care Belgrade. Archives of Emergency Medicine and Intensive Care. 2018; 1(1): 34-37.

Copyright: (C) 2018 Plazinic Djurdja M., Zivanovic Slavoljub R. This is an open access article distributed under the Creative Commons Attribution License, which permits unrestricted use, distribution, and reproduction in any medium, provided the original work is properly cited. 